Südosteuropa - Studien · Band 12

(eBook - Digi20-Retro)

\title{
Rudolf Bićanić
}

\section{Jugoslawiens Stellung \\ in der Weltwirtschaft und das \\ Auslandskapital in Jugoslawien}

Verlag Otto Sagner München - Berlin - Washington D.C.

Digitalisiert im Rahmen der Kooperation mit dem DFG-Projekt „Digi20“

der Bayerischen Staatsbibliothek, München. OCR-Bearbeitung und Erstellung des eBooks durch den Verlag Otto Sagner:

http://verlag.kubon-sagner.de

( bei Verlag Otto Sagner. Eine Verwertung oder Weitergabe der Texte und Abbildungen, insbesondere durch Vervielfältigung, ist ohne vorherige schriftliche Genehmigung des Verlages unzulässig.

«Verlag Otto Sagner» ist ein Imprint der Kubon \& Sagner GmbH. 
61.42<smiles>C1CC1</smiles>

\section{S ÜDOSTEU R O PA - STU DIEN}

im Namen der Südosteuropa-Gesellschaft herausgegeben von WALTER ALTHAMMER

Rudolf Bićanić

\section{Jugoslawiens Stellung in der Weltwirtschaft und das Auslandskapital in Jugoslawien}

Festvortrag

gehalten am 2. Dezember 1967 in München auf der Jahreshauptversammlung der Südosteuropa-Gesellschaft 


\section{SÜDOSTEUROPA-GESELLSCHAFT}

8 München 22, Widenmaryerstr. 49 - Tel. 294402

\section{PRÄSIDIUM}

Präsident: $\quad$ Dr. Walter Althammer, MdB, Augsburg

Vizepräsidenten: Prof.Dr. Hermann Gross, München

Alfred Hönig, Salzburg

Dr. Günther Müller, MdB, München

Schatzmeister: Bankdirektor Dr. Hamilkar Hofmann, München

Beisitzer: Dr. Mathias Bernath, München

Redakteur Hans Hartl, Starnberg

Prof. Dr. Hans-Joachim Kissling, München

Landeshauptmann Dr. Ing. Hans Lechner, Salzburg

Bankdirektor Dr. Ernst Lederer, Frankfurt

Prof. Dr. Alois Schmaus, München

Dr. Emanuel Turczynski, München

WISSENSCHAFTLICHER BEIRAT

Vorsitzender: Prof.Dr. Alois Schmaus, München

Stellv. Vorsitzender: Prof. Dr. Hermann Gross, München

Sekretär: Dr. Emanuel Turczynski, München 
$\approx \Rightarrow d$

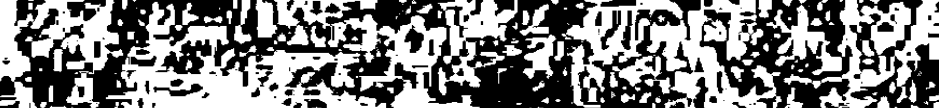

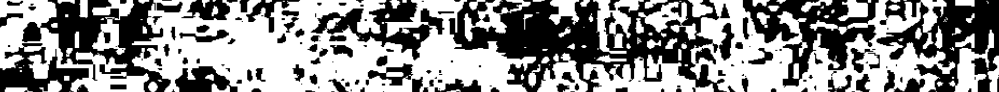

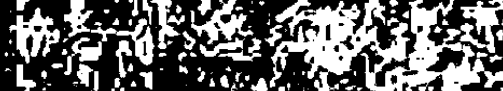

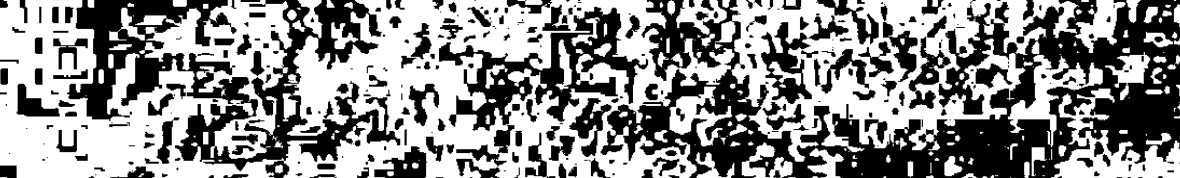

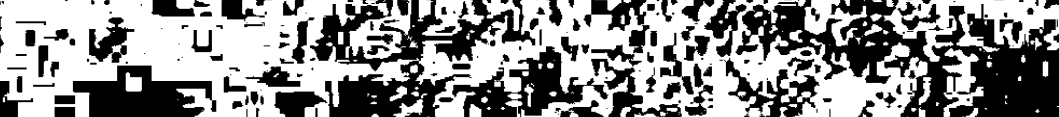

int

A

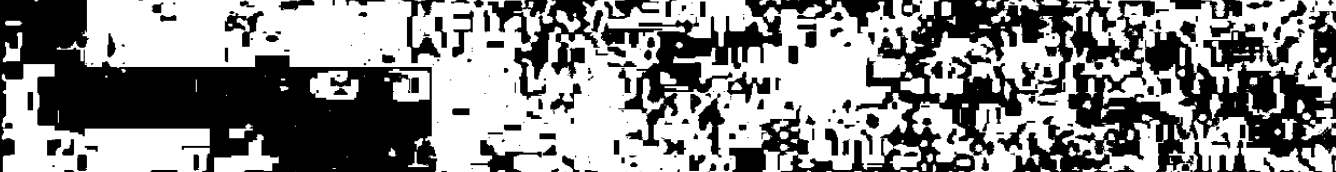

ror

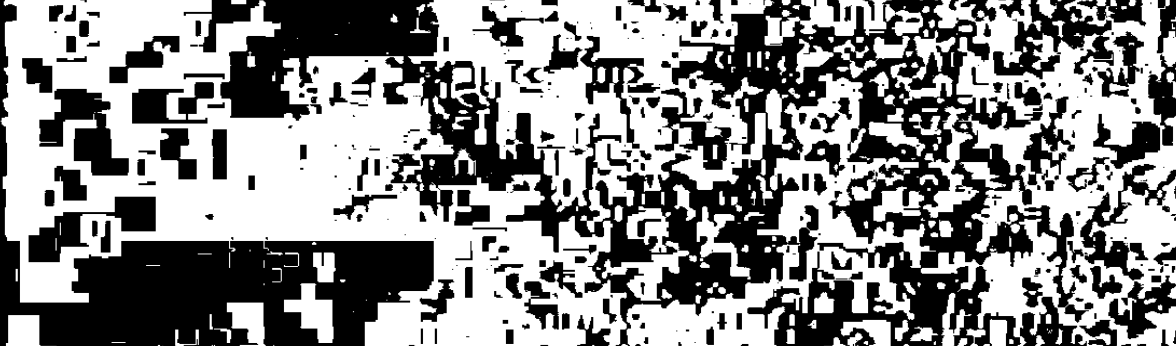

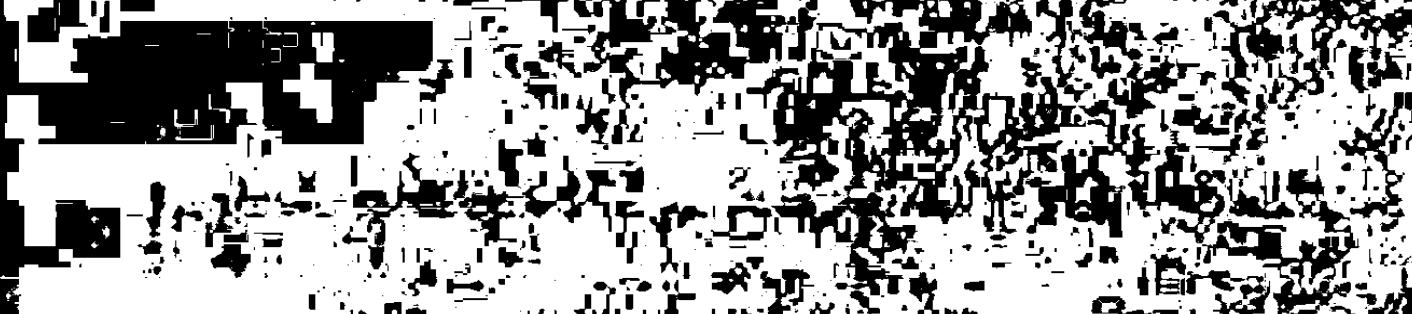

int $x^{2}$

is or

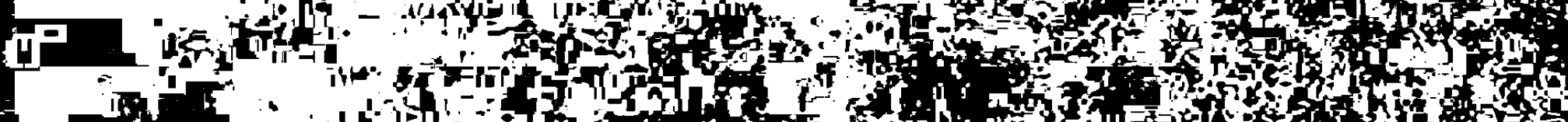

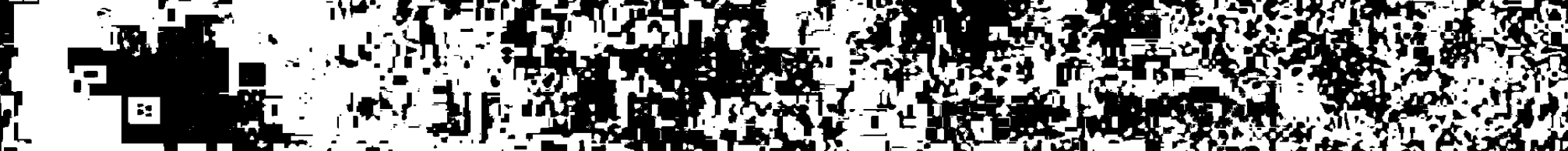

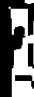

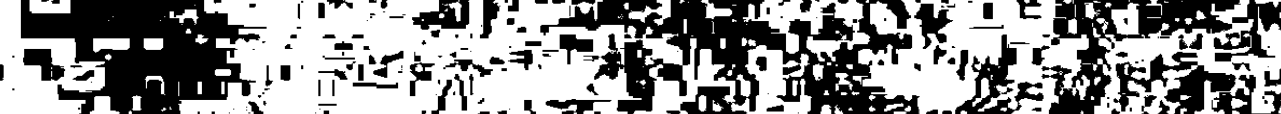

Tor

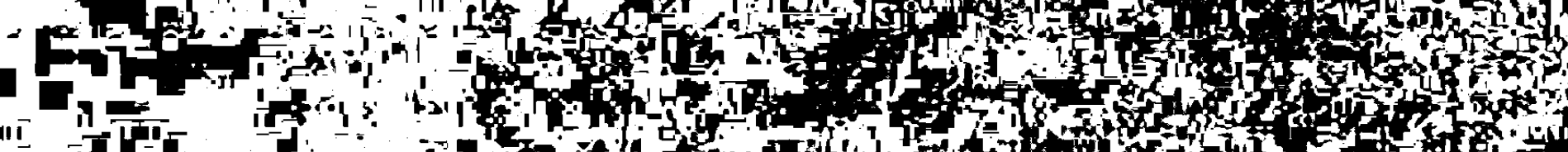

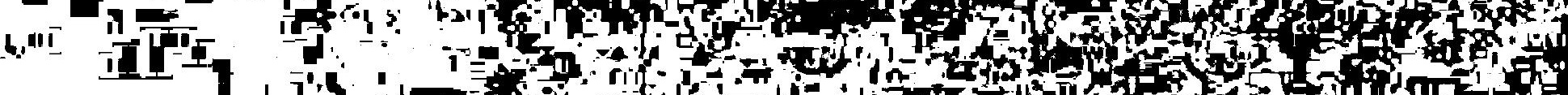

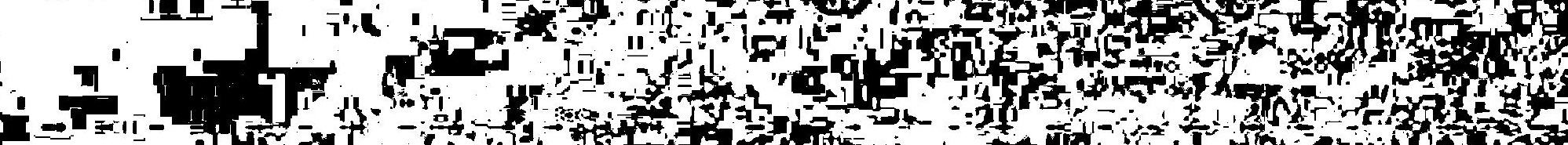

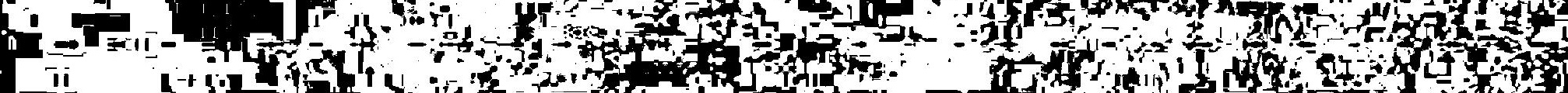
and I I , I

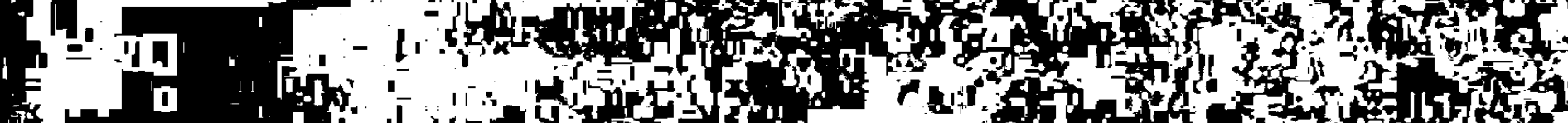

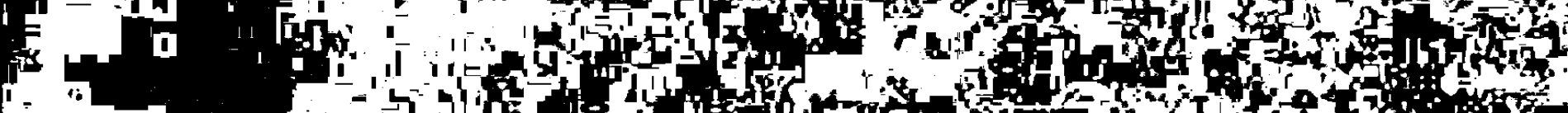

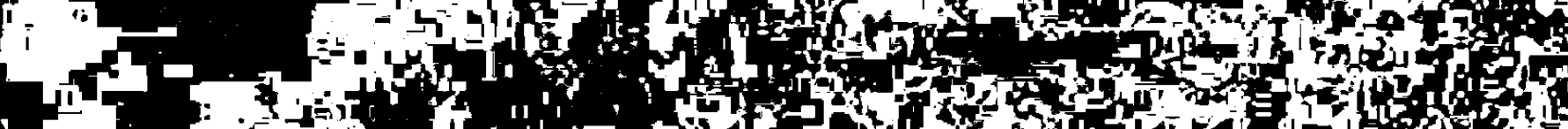
1. 25r

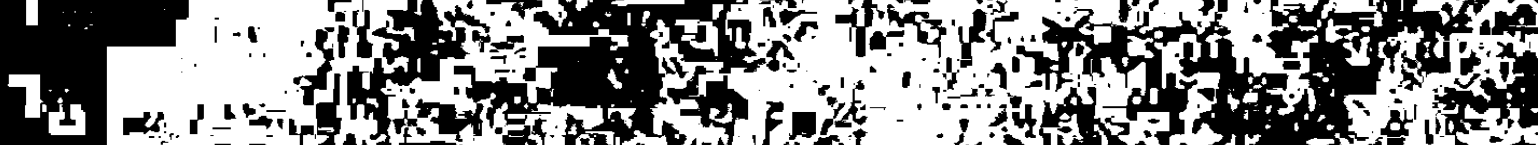

TH

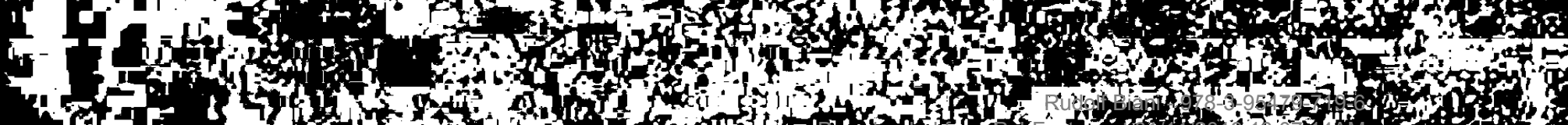

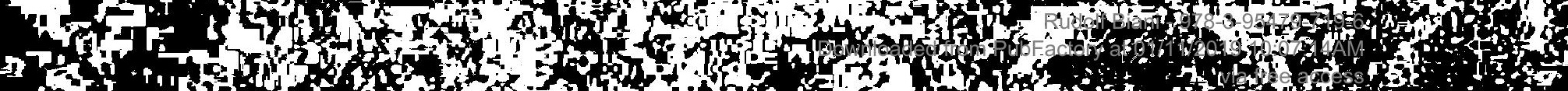


$z 61,42$

\title{
S ÜD OSTE U RO PA - S T U DIEN
}

im Namen der Südosteuropa-Gesellschaft herausgegeben von WALTER ALTHAMMER

\author{
Rudolf Bićanić
}

\section{Jugoslawiens Stellung in der Weltwirtschaft und das Auslandskapital in Jugoslawien}

\section{Festvortrag}

gehalten am 2. Dezember 1967 in München auf der Jahreshauptversammlung der Südosteuropa-Gesellschaft 
Übersetzung: Dipl.-Volksw. Johann Hawlowitsch, München Redaktion: Dr. Hanna Gülich-Bielenberg, Kiel 
Der Vortrag, den Professor Dr. Rudolf Bićanić am 2. Dezember 1967 auf der Jahreshauptversammlung der Südosteuropa-Gesellschaft in München gehalten hat, erscheint post mortem. Ein allzu früher Tod riß den hervorragenden Gelehrten am 9.7.1968 in Zagreb aus seinem reichen Schaffen heraus.

Rudolf Bićcanić, der 1905 in Bjelovar/Kroatien geboren wurde, war Professor für Wirtschaftswissenschaften an der Rechtswissenschaftlichen Fakultät der Universität Zagreb.

Sein wissenschaftlicher Werdegang sei kurz skizziert: 1925 Certificate d' Etudes der Ecole des Hautes Etudes Commerciales, Paris, - 1931 Promotion an der Rechtswissenschaftlichen Fakultät der Universität Zagreb, 1936-40 Direktor des Instituts für agrarwirtschaftliche Forschung und Nationalökonomie, Zagreb, - 1940-46 Vizegouverneur der Jugoslawischen Nationalbank und Generaldirektor für Außenhandel in Großbritannien, 1946 Lehrstuhl für angewandte Wirtschaftswissenschaften an der Rechtswissenschaftlichen Fakultät der Universität Zagreb, - 1952-61 Direktor des Instituts für Wirtschaftswissenschaften an der Universität Zagreb. Als Gastprofessor lehrte er 1958 an der Universität Texas, $1962-65$ am Institut für Sozialwissenschaften in Den Haag/Niederlande und 1966-67 am Fellow Center for Advanced Studies in the Behavioral Sciences in Stanford/ Californien.

Rudolf Bićanić galt als eine der führenden und bedeutendsten Persönlichkeiten des wissenschaftlichen Lebens Jugoslawiens. Er war international anerkannt.

Seine Forschung und seine zahlreichen Schriften umfassen Theorie und Praxis der wirtschaftlichen Entwicklung, der Wirtschaftsplanung und Wirtschaftslenkung, Typologie der Planung, Außenwirtschaft sowie Wirtschaftsstruktur und Wirtschaftspolitik, im besonderen Agrarpolitik, in Jugoslawien.

Bićanić war in seiner theoretischen Forschung und Lehre stark mit der Wirklichkeit verbunden. Er war auch aktiv im wirtschaftlich/politischen Leben Jugoslawiens tätig. In seiner klaren und unabhängigen Denkart, in seinem Streben nach wissenschaftlicher Wahrheit diente er dem Ziel, die Völker einander näher zu bringen und das gegenseitige Verständnis zu fördern.

Möge die Veröffentlichung seines letzten Vortrages in der SüdosteuropaGesellschaft auch diesem Ziel dienen.

Augsburg, im November 1968

Dr. Walter Althammer

Präsident der Südosteuropa-

Gesellschaft 


\title{
Jugoslawiens Stellung in der Weltwirtschaft und das Auslandskapital in Jugoslawien
}

\author{
A u B nhandel und A B enhandelspolitik
}

Die offizielle jugoslawische Außenhandelsdoktrin wurde nach 1945 aus der alten, vor dem Krieg verfolgten Handelspolitik entwickelt. Diese beinhaltete eine Abschließung vom Weltmarkt bei gleichzeitiger Entwicklung der Volkswirtschaft durch beschleunigten Aufbau der Industrie und verfolgte das Ziel einer nationalen Autarkie.

Die sozialistische Revolution fügte ein neues Element hinzu: die zentralgeleitete autoritäre Planwirtschaft mit einem (de facto) staatlichen Außenhandelsmonopol. Es wurde eine wirksame Schranke zwischen der im Inland betriebenen Planwirtschaft und der Weltwirtschaft errichtet, zwischen den inländischen geplanten Preisen und den Weltmarktpreisen, den inländischen Planauflagen (die administrativ bestimmt wurden) und den vertraglichen Beziehungen zu ausländischen Firmen, wobei jede Transaktion vom Außenhandelsministerium genehmigt werden mußte. Es war dies ein Handel, der streng auf bestimmte Märkte ausgerichtet war. Der Zahlungsverkehr wurde in einem bilateralen Clearingsystem abgewickelt.

Diese autarkiepolitische Konzeption wurde in zweierlei Hinsicht durchbrochen: Erstens durch die beschleunigte Industrialisierungspolitik unter der Vorstellung, man könne die industrielle Revolution im Lande mit Hilfe von einem oder zwei Fünfjahresplänen durchsetzen, - die Ausfuhr wurde in den Dienst dieser Politik gestellt, und es wurden Ausfuhrüberschüsse bestimmt bzw. gebildet, um mit ihnen die Einfuhr von Gütern hoher Dringlichkeit zu bezahlen -; zweitens durch die nicht aus dem Handelsverkehr stammenden Teile der Einfuhr, wie z.B. die Hilfe seitens der UNRRA, Reparationszahlungen usw.

Der Außenhandel stand unter einem starken ideologischen Einfluß der ersten Tage nachrevolutionärer Begeisterung, auf die das sow jetische Prinzip "Freundschaft ist Freundschaft und Handel ist Handel" wie eine eiskalte und ernüchternde Dusche wirkte ${ }^{2}$. Es folgte (1948-55) die Wirtschaftsblockade seitens der sozialistischen Länder, sowie die völlige Umorientierung des Außenhandels von Ost nach West; zu der auch die Hilfe der USA, des Vereinigten Königreichs und Frankreichs entscheidend beigetragen hat.

Vgl. M. Popović, O ekonomskim odnosima izmedju socijalističkih drŽava (Über wirtschaftliche Beziehungen zwischen den sozialistischen Staaten), Beograd 1949, S. 34 . 
Das System des administrativen Sozialismus wurde 1952 aufgegeben und ein neues Wirtschaftssystem unter den Bedingungen der Arbeiterselbstverwaltung eingeführt. Im Bereich des Außenhandels bedeutete dies eine Kommerzialisierung der geschäftlichen Transaktionen mit dem Ausland, d.h. die sozialistischen Unternehmungen konnten in eigener Verantwortung Geschäfte mit dem Ausland abschließen'.

Die Autarkie als das oberste Ziel der Außenhandelstätigkeit blieb auf der Makro-Ebene bestehen und wurde auf der Mikro-Ebene (Unternehmungen) nach und nach abgebaut. Bezüglich des Warenverkehrs erhielten die Pläne mehr indikativen als direktiven Charakter, wobei die operativen Pläne von den Unternehmungen selbst beschlossen werden konnten. Das Wirksamwerden marktwirtschaftlicher Mechanismen im Inland erleichterte den Handel mit dem Ausland, obwohl für bestimmte Güter, insbesondere für gewisse Rohstoffe, das System der zentralen Verteilung aufrechterhalten wurde. Auch die Verteilung der durch den Export eingenommenen Devisen wurde arbiträr durch die Nationalbank vorgenommen, die selber eine streng zentralisierte staatliche Anstalt war. Es wurde der Grundsatz einer Öffnung der Volkswirtschaft zum Weltmarkt proklamiert (1952), jedoch bezog sich dies nur auf einen kleinen Bereich des Handels.

Die Herstellung der Autonomie bei den Unternehmungen, die nun freie Initiative hatten, führte zu einer Ausdehnung des Kreises der Handelspartner von 63 (1956) auf 120 (1966) Länder, wozu auch die Außenpolitik der Nichtverpflichtung viel beigetragen hat. Es wurden große Erwartungen in die Entfaltung des Außenhandels mit Entwicklungsländern gesetzt, jedoch war der Erfolg nur begrenzt. In bedeutendem Ausmaß wurde auch das Sortiment an Ein- und Ausfuhrwaren erweitert, doch hatte die Diversifizierung der Ausfuhr nicht immer den gewünschten Effekt. Sie wurde im Bereich des Verrechnungsverkehrs von einer Politik gespaltener Wechselkurse begleitet, die schließlich dazu führte, daß man ökonomisch nicht genau ermitteln konnte, mit welchem Erfolg man Außenhandel trieb.

Die Passivităt der Zahlungsbilanz, eine unmittelbare Folge des begrenzten Exports, war letzten Endes eine Auswirkung des beschleunigten wirtschaftlichen Wachstums im Inland. Dieses Tempo des Wachstums erfor-

1946 waren lediglich 9 Unternehmungen registriert, die sich im Außenhandel betätigten. 1947 erhöhte sich ihre Zahl auf 154, darunter 60 Generaldirektionen des Ministeriums. Im Zuge des Übergangs vom administrativ geleiteten zum kommerzialisierten Außenhandel stieg die Zahl der Außenhandelsunternehmungen von 154 auf 320, wobei die Direktionen vollkommen abgeschafft wurden. 1962 gab es 485 Außenhandelsunternehmungen. In der Folgezeit schwankte ihre Zahl zwischen 377 und 511. Die Wirtschaftsreform von 1965 führte zu einer weiteren Konzentration der Außenhandelsunternehmungen, so daß die Anzahl der ausschließlich im Außenhandel tätigen Unternehmungen sich von 204 auf 141 verringerte. Sie beschäftigten 28.058 Personen und wiesen ein Kapital von 275 Mill. US Dollar sowie einen Umsatz von 1.940 Mill. US Dollar aus. 
derte eine stetige Vergrößerung der Einfuhr. Die Ausfuhr aber wurde infolge der steigenden inländischen Nachfrage gehemmt. Die inflatorische Entwicklung unterstützte den Absatz ausländischer Wirtschaftsprodukte auf inländischen Märkten. Durch die Währungspolitik der multiplen Wechselkurse wurde die Ausfuhr behindert sowie die Verbindung zwischen Einund Ausfuhr aufgehoben, die ein freier Markt sonst gewährleistet hätte.

In dieser Zeit (bis 1964) erhöhte sich der Außenhandel auf 1.323 Mill. Dollar Einfuhr und $893 \mathrm{Mill}$. Dollar Ausfuhr. Vor dem Zweiten Weltkrieg beliefen sich die Ein- und Ausfuhren auf je 100 Mill. Dollar; ein eindrucksvoller Vergleich! (Siehe hierzu Tabelle 1 und 2.)

Tab. 1: Ausfuhr, Einfuhr und Saldo der Handelsbilanz Jugoslawiens $1947-1967$

(Mill. neue Dinar; 1 US $\$=12,50$ neue Dinar)

Jahr

Ausfuhr

Einfuhr

Saldo der

Handelsbilanz

\begin{tabular}{lrrr}
\hline 1947 & 2046 & 2076 & - \\
1948 & 3712 & 3831 & -119 \\
1949 & 2484 & 3685 & -1201 \\
1950 & 1929 & 2883 & - \\
1951 & 2234 & 4797 & -2563 \\
1952 & 3082 & 4664 & -1582 \\
1953 & 2325 & 4941 & -2616 \\
1954 & 3005 & 4242 & -1237 \\
1955 & 3207 & 5512 & -2305 \\
1956 & 4042 & 5927 & -1885 \\
1957 & 4939 & 8266 & -3327 \\
1958 & 5517 & 8563 & -3046 \\
1959 & 5958 & 8590 & -2632 \\
1960 & 7077 & 10330 & -3253 \\
1961 & 7111 & 11379 & -4268 \\
1962 & 8631 & 11097 & -2466 \\
1963 & 9879 & 13208 & -3329 \\
1964 & 11164 & 16540 & -5376 \\
1965 & 13644 & 16099 & -2455 \\
1966 & 15251 & 19693 & -4442 \\
1967 & 15661 & 21345 & -5684
\end{tabular}

Quelle: Statistika spoljne trgovine Jugoslavije 1966 (Statistik des jugoslawischen Außenhandels 1966), Beograd 1967, S. 15; "Indeks", Nr.2, Beograd 1968. 
Tab. 2: Warenstruktur der jugoslawischen Exporte und Importe nach Warengruppen 1952-1967 (in v.H.)

\begin{tabular}{|c|c|c|c|c|c|c|}
\hline \multirow{2}{*}{$\operatorname{Jah} \mathbf{r}$} & \multicolumn{3}{|c|}{ Ausfuhr ${ }^{a}$} & \multicolumn{3}{|c|}{ Einfuhr ${ }^{\mathbf{a}}$} \\
\hline & A & B & $\mathrm{C}$ & A & $\mathbf{B}$ & C \\
\hline $\begin{array}{l}1952 \\
1953 \\
1954\end{array}$ & $\begin{array}{l}87,9 \\
77,0 \\
68,9\end{array}$ & $\begin{array}{l}0,8 \\
3,2 \\
4,8\end{array}$ & $\begin{array}{l}11,3 \\
19,8 \\
26,3\end{array}$ & $\begin{array}{l}52,0 \\
56,6 \\
60,1\end{array}$ & $\begin{array}{l}37,7 \\
37,9 \\
31,5\end{array}$ & $\begin{array}{r}10,3 \\
7,7 \\
8,4\end{array}$ \\
\hline $\begin{array}{l}1955 \\
1956 \\
1957 \\
1958 \\
1959\end{array}$ & $\begin{array}{l}72,3 \\
53,9 \\
52,6 \\
44,4 \\
47,2\end{array}$ & $\begin{array}{r}3,5 \\
3,1 \\
5,0 \\
8,1 \\
11,8\end{array}$ & $\begin{array}{l}24,2 \\
43,0 \\
42,4 \\
47,5 \\
41,0\end{array}$ & $\begin{array}{l}59,0 \\
51,0 \\
55,2 \\
52,1 \\
56,2\end{array}$ & $\begin{array}{l}29,6 \\
14,0 \\
18,2 \\
23,6 \\
19,8\end{array}$ & $\begin{array}{l}11,4 \\
35,0 \\
26,6 \\
24,3 \\
24,0\end{array}$ \\
\hline $\begin{array}{l}1960 \\
1961 \\
1962 \\
1963 \\
1964\end{array}$ & $\begin{array}{l}45,5 \\
44,0 \\
43,5 \\
41,7 \\
42,1\end{array}$ & $\begin{array}{r}9,7 \\
10,5 \\
17,6 \\
13,9 \\
11,7\end{array}$ & $\begin{array}{l}44,8 \\
45,5 \\
38,9 \\
44,4 \\
46,2\end{array}$ & $\begin{array}{l}56,6 \\
52,3 \\
54,6 \\
57,7 \\
62,5\end{array}$ & $\begin{array}{l}28,6 \\
28,2 \\
25,2 \\
20,0 \\
21,6\end{array}$ & $\begin{array}{l}14,8 \\
19,5 \\
20,2 \\
22,3 \\
15,9\end{array}$ \\
\hline $\begin{array}{l}1965 \\
1966 \\
1967\end{array}$ & $\begin{array}{l}42,0 \\
43,0 \\
48,1\end{array}$ & $\begin{array}{l}17,2 \\
18,3 \\
14,1\end{array}$ & $\begin{array}{l}40,8 \\
38,7 \\
37,8\end{array}$ & $\begin{array}{l}62,1 \\
57,5 \\
57,1\end{array}$ & $\begin{array}{l}19,7 \\
21,8 \\
21,7\end{array}$ & $\begin{array}{l}18,2 \\
20,7 \\
21,2\end{array}$ \\
\hline
\end{tabular}

Quelle: Statistika spoljne trgovine Jugoslavije (Statistik des jugoslawischen Außenhandels), Beograd, versch. Jahrgänge.

Jugoslawiens Außenhandel ist am Welthandel noch immer mit weniger als 5 Promille beteiligt. Dem Umfang nach hat er aber das Außenhandelsvolumen von Argentinien, Mexiko und Venezuela erreicht und liegt etwas unter dem Österreichs und etwas über dem Bulgariens.

Die Ausfuhr je Einwohner betrug 1937: 8 Dollar, 1956: 18 Dollar und erreichte 1966: 66 Dollar. Die entsprechenden Zahlen für die Einfuhr waren 1937: 7, 1957: 27 und 1966: 81 Dollar je Einwohner.

Der durch die Autarkiepolitik gesetzte Rahmen wurde zu eng für die weitere wirtschaftliche Entwicklung des Landes. Die überaus schnelle Entwicklung führte zu einem Gegensatz zwischen der Anderung der Außenhandelsstruktur in Richtung Autarkie einerseits und der Wirtschaftlichkeit dieser Strukturänderungen sowie der Rentabilität des Außenhandels andererseits. Das schnelle Wachstum mit einer der höchsten Zuwachsraten 
in der Welt von $8,5 \mathrm{v} . \mathrm{H}$. erforderte vielmehr eine Überprüfung seiner Effizienz an internationalen Maßstäben, d.h. am Weltmarkt mit Hilfe von Konkurrenzpreisen.

Im Gegensatz zur alten autarkieorientierten Auffassung, daß die wirtschaftliche Entwicklung zur Unabhängigkeit von Auslandsmärkten führe, d.h. der Güteraustausch mit dem Ausland abnähme, wurde es nun offenkundig, daß die wirtschaftliche Entwicklung zu einer Zunahme des Austausches führen muß und die nationale Unabhängigkeit auf anderen Gebieten anzustreben sei, - d.h. in der Äquivalenz des Austausches. Dafür war aber eine feste Bezugsgröße im inländischen Geldsystem, den Weltmarktpreisen und der Konvertibilităt der Währung notwendig. Mit anderen Worten: man mußte die Illusion einer nationalen Autarkiepolitik in einem Wirtschaftsraum mit 20 Millionen Menschen und einem Nationaleinkommen pro Kopf der Bevölkerung von 500 Dollar überwinden.

Im Zuge der 1965 durchgeführten Wirtschaftsreformen zeichnete sich ziemlich deutlich eine Politik der bewußten und beabsichtigten Einschaltung in die internationale Arbeitsteilung ab. Dieses Ziel soll nicht nur mittels handelspolitischer Maßnahmen erreicht werden, sondern durch einen Einbau der jugoslawischen Volkswirtschaft in die internationale Arbeitsteilung.

Die Neuorientierung in der Außenwirtschaftspolitik ${ }^{2}$ wurde durch folgende

1 Im ersten Fünfjahresplan (1947-51) wurden dem Außenhandel keine Aufgaben zugewiesen (mit Ausnahme in den konkreten operativen Plänen). Vom Außenhandel war nur im negativen Sinne die Rede, nämlich, daß es "über einen Zeitraum von mehreren Jahren unmöglich ist, den Wiederaufbau des Staates ausschließlich im Wege von Importen notwendiger Maschinen aus dem Ausland zu bewerkstelligen". Über die Ein- und Ausfuhrpläne wurde nichts veröffentlicht.

Vgl. Petogodisnji plan razvitka narodne privrede FNRJ 1947-51 (Fünfahresplan der Entwicklung der Volkswirtschaft der FVR Jugoslawien 1947-51), Beograd 1947, S. 8 .

Nach 1952 wurden in den Plänen lediglich die globalen makro-ökonomischen Proportionen des Außenhandels bestimmt, die als Richtlinien bei der Genehmigung der von den Unternehmungen selbst erstellten Pläne dienten.

Der Fünfjahresplan 1957-61 widmete den Außenhandelsproblemen große Aufmerksamkeit. Der Außenhandel stand an zweiter Stelle unter den fün wichtigsten Planzielen und sollte $z u$ einer beschleunigten wirtschaftlichen Entwicklung des Landes beitragen. Zum Zwecke einer Verringerung des Handelsbilanzdefizits sowie eines Ausbaues der Handelsbeziehungen mit dem Ausland sollte die Ausfuhr schneller als die Einfuhr wachsen. Es wurden ferner die Bereiche angeführt, in denen der Außenhandel und die Änderung seiner Struktur besonders gefördert sowie ein Abbau der Auslandsverschuldung erreicht werden sollten.

Vgl. Društveni plan privrednog razvoja Jugoslavije 1957-61 (Gesellschaftsplan der Wirtschaftsentwicklung Jugoslawiens 1957-61), Beograd 1957, S. 145-151.

Fortsetzung siehe nächste Seite 
- Die Teilung in ein inländisches und ausländisches Preissystem führte zu immer größeren Preisdisproportionen, was eine Subventionierung der Ein- und Ausfuhr in einem Ausmaß erforderte, das für den Bundeshaushalt unerträglich wurde (300 Milliarden Dinar) und einer Dezentralisierungs- und Föderalisierungspolitik im Lande selbst entgegenstand.

- Gespaltene Wechselkurse führten dazu, daß man nicht feststellen konnte, inwieweit der Austausch von Gütern und Dienstleistungen mit dem Ausland rationell war. Deshalb wurde 1965 ein Einheitskurs auf 1 US Dollar = 12, 50 neue Dinar festgesetzt.

- Das Zahlungsbilanzdefizit ist seit 1945 eine permanente Erscheinung im jugoslawischen Außenhandel. Man mußte es durch eine verstärkte Stimulierung der Ausfuhr beseitigen, was mit administrativen Maßnahmen allein nicht gelang, sondern nur durch eine Liberalisierung des Außenhandels erreicht werden konnte. Die Liberalisierung nach den Grundsătzen des GATT führte zu einem Abbau der Zölle, die von 23 v.H. auf durchschnittlich 11 v.H. reduziert wurden. Die Liberalisierung des Güterverkehrs zog auch eine Liberalisierung des Geldverkehrs nach sich und gegenwărtig verfolgt man das Ziel, die Konvertibilităt des Dinar herzustellen, sobald die Devisenreserve auf 500 Millionen Dollar angewachsen ist. (Vgl. Tabelle 3 und 4, s. Seite 11 und 12.)

Die Verschuldung gegenüber dem Ausland wurde zu einem ernsten Problem; 1965 mußten 31 v. H. aller Devisenmittel für die Rückzahlung ausländischer Schulden (einschließlich der Zinsbelastung) verwendet werden. Die Politik kurzfristiger und kommerzieller Verschuldung zur Deckung des Handelsbilanzdefizits konnte nicht ins Grenzenlose ausgedehnt werden ${ }^{2}$.

- Die Notwendigkeit einer verstärkten Ausfuhr und Substitution der Einfuhr durch einheimische Erzeugnisse führte zum Import von Produktionslizenzen, vornehmlich im Maschinenbau und für chemische Verfahren.

Fortsetzung der Fußnote 1 von Vorseite:

Im dritten Fünfjahresplan 1961-65 wurde die Herstellung des Zahlungsbilanzgleichgewichts eingeplant, ein Ziel das unter den Planzielen an dritter Stelle stand. Im laufenden Plan für 1966-70 erhielten die außenwirtschaftlichen Planziele Einschaltung in die weltwirtschaftliche Arbeitsteilung und Herstellung der Konvertibilităt des Dinars den sechsten Platz unter den sieben Planzielen.

1 Die Verschuldung gegenüber dem Ausland betrug 1956: 415 Mill. Dollar und erreichte 1966 eine Höhe von 1096 Mill. Dollar. Vgl. I. Zuvela, Medjunarodni ekonomski odnosi (Internationale Wirtschaftsbeziehungen), Zagreb 1967, S. 517 . 
Tab. 3: Zugang und Abgang an ausländischen Zahlungsmitteln 1965 und 1966

(Mill. neue Dinar; 1 US $\$=12,50$ neue Dinar)

I. Zugang

Insgesamt

Ausfuhr

Verkehr und Versicherung

Nicht aus dem Warenverkehr stammender Zugang:

davon Auswandererüberweisungen $^{2}$ Fremdenverkehr Unterhaltung diplomat. Vertretungen und ähnl. Zugänge

Sonstiges

II. Abgang

Insgesamt

Einfuhr

Verkehr und Versicherung

Nicht aus dem Warenverkehr stammender Abgang

Sonstiges

III. Saldo (I - II)
16828

13022

1651

2069
3010

19406

14362

1965
1190

1459

1014

329

361

69

20920

16252

17830

1145

1362

839

554

889

\begin{tabular}{r|c}
730 & 889 \\
\hline-1853 & -1514 \\
\hline
\end{tabular}

a Einschließlich Überweisungen jugoslawischer Gastarbeiter

Quelle: Statisticki bilten Službe Druß̌tvenog Knjigovodstva (Statist. Bulletin des Gesell schaftlichen Buchführungsdienstes), Beograd, H. 1 (1968), S. 78. 
Tab. 4: Finanzielle Abrechnung mit dem Ausland 1965 und 1966 (Mill. neue Dinar; 1 US $\$=12,50$ neue Dinar)

I. Mittelinanspruchnahme und Verschuldung Zugang

Insgesamt

5541

Verminderung der Devisenreserven

Erhöhung der Clearingverbindlichkeiten

Mittelinanspruchnahme:

Anleihen und Kredite kurzfristige Kredite Reparationen und Sonderansprüche Sonderanleihen

Subventionen und Kreditvorschüsse

II. Mittelerhöhung und Schuldenrückzahlung Abgang

Insgesamt

Erhöhung der Devisenreserven Verminderung der Clearingverbindlichkeiten

Rückzahlungen:

Anleihen und Kredite kurzfristige Kredite nationalisiertes Eigentum

Sonstiges

III. Saldo (I - II )
3688

4551 671

5290
5166

8

4931

2822

Quelle: Statistički bilten Službe DruStvenog Knjigovodstva (Statist. Bulletin des Gesellschaftlichen Buchführungsdienstes), Beograd, H. 1 (1968), S. 79. 
Zu Hunderten übernahm man solche Lizenzen. Doch hatte auch Jugoslawien eine bestimmte Anzahl von eigenen Lizenzen, die es an ausländische Interessenten veräußern konnte.

Eine bedeutende Position in der jugoslawischen Zahlungsbilanz nehmen die unsichtbaren Einnahmen ein, welche die wirtschaftliche Entwicklung des Landes entscheidend mitbestimmen. Hier seien nur die wichtigsten genannt:

Der Tourismus steuert heute direkt oder indirekt $z u$ den Einnahmen der Zahlungsbilanz fast 200 Millionen Dollar bei; die Überweisungen von Auswanderern und Gastarbeitern in Westeuropa liegen bei einem Betrag von über 100 Millionen Dollar; schließlich wären noch die Einnahmen aus den Verkehrsleistungen, insbesondere den Leistungen der Handelsmarine zu nennen; die Arbeiten jugoslawischer Unternehmungen im Ausland erlangen gleichfalls immer größere Bedeutung: heute liegen Vertragsabschlüsse von mehreren 100 Millionen Dollar vor, und zwar nicht nur mit unterentwickelten, sondern auch mit europäischen Ländern, wie der Bundesrepublik Deutschland und der Tschechoslowakei.

Ein besonderes Problem dieser Entwicklung liegt darin, daß ein großer Teil der im Ausland tätigen jugoslawischen Arbeiter eines Tages heimkehren wird, für den dann ausreichende Beschäftigungsmöglichkeiten geschaffen werden müssen.

Weitere Schwierigkeiten in der Entwicklung des Außenhandels sind in seiner ungleichmäßigen regionalen Ausrichtung zu suchen: die Handelsbilanz mit den westlichen Ländern ist stark passiv, mit den östlichen jedoch aktiv. Die Tabelle 5 zeigt die Entwicklung in den letzten beiden Jahren. Die

Tab. 5: Der jugoslawische Außenhandel nach Wirtschaftsraumen 1962, 1966 und 1967 (Mill. ne ue Dinar und v.H.)

\begin{tabular}{|c|c|c|c|c|c|c|c|c|c|c|}
\hline & & \multicolumn{3}{|c|}{ Ausfuhr } & \multicolumn{3}{|c|}{ Einfuhr } & \multicolumn{3}{|c|}{ Saldo } \\
\hline & & 1962 & 1966 & 1967 & 1962 & 1966 & 1967 & 1962 & 1966 & 1967 \\
\hline EFTA & $\begin{array}{l}\text { Mill. Dinar } \\
\text { v.H. }\end{array}$ & $\begin{array}{l}1280 \\
14,9\end{array}$ & $\begin{array}{l}1666 \\
11,8\end{array}$ & $\begin{array}{l}1721 \\
10,3\end{array}$ & $\begin{array}{l}1396 \\
12,6\end{array}$ & $\begin{array}{l}2185 \\
11,1\end{array}$ & $\begin{array}{l}2651 \\
12,4\end{array}$ & -116 & -519 & -930 \\
\hline EWG & $\begin{array}{l}\text { Mill. Dinar } \\
\text { v.H. }\end{array}$ & $\begin{array}{l}2482 \\
28,8\end{array}$ & $\begin{array}{l}4617 \\
30,2\end{array}$ & $\begin{array}{l}5020 \\
30,2\end{array}$ & $\begin{array}{l}3360 \\
30,4\end{array}$ & $\begin{array}{l}5412 \\
27,5\end{array}$ & $\begin{array}{l}6593 \\
31,0\end{array}$ & -878 & -795 & -1573 \\
\hline COMECON & $\begin{array}{l}\text { Mill. Dinar } \\
\text { v. H. }\end{array}$ & $\begin{array}{l}2087 \\
24,3\end{array}$ & $\begin{array}{l}5575 \\
36,6\end{array}$ & $\begin{array}{l}5670 \\
34,0\end{array}$ & $\begin{array}{l}2352 \\
21,2\end{array}$ & $\begin{array}{l}6152 \\
31,2\end{array}$ & $\begin{array}{l}5532 \\
25,9\end{array}$ & -265 & -577 & 138 \\
\hline
\end{tabular}

Quelle: Statistika spoljne trgovine Jugoslavije (Statistik des jugoslawischen AuBenhandels), Beograd, versch. Jahrgänge. 
Forcierung der Ausfuhr in die osteuropäischen (sozialistischen) Länder, u. a. unterstützt durch relativ hohe Preise für jugoslawische Produkte in diesen Ländern, wird paralysiert durch die weit geringeren Einfuhren aus diesem Raum. Das ist hauptsächlich auf die starre Handelspolitik, das dürftige Sortiment und die ungenügende Qualität der Exportgüter, insbesondere der Konsumgüter, der östlichen Länder zurückzuführen ${ }^{1}$.

Die jugoslawische Wirtschaftspolitik steht nun vor der Entscheidung, wie die Folgen der beabsichtigten stärkeren Einschaltung in die internationale A rbeitsteilung auf die einzelnen Wirtschaftszweige aufgeteilt werden sollen. Die Richtung dieser Einschaltung wurde in allgemeinen Zügen im Fünfjahresplan 1966-70 festgelegt. Dort wurden auch die Produktionszweige genannt, die einen komperativen Vorteil gegenüber dem Weltmarkt besitzen $^{2}$. Dies sind: Produktion und Verarbeitung von Buntmetallen: Kupfer, Blei und Bauxit; Anlagenherstellung, Schiffbau sowie langlebige Konsumgüter aus Metall; landwirtschaftliche Erzeugung, insbesondere von Weizen, Mais, Fleisch und einigen Industriekulturen; mechanische und chemische Holzverarbeitung; Fremdenverkehr; internationaler Durchgangsverkehr; Stahlproduktion; Produktion zeitgemäßer Baumaterialien; Erdöl und Erdgas; Kunstdünger; Kunststoffe; Textilkonfektion und Ledererzeugnisse ${ }^{3}$.

Eine Wirtschaftspolitik, die auf eine Öffnung der Volkswirtschaft zum Weltmarkt ausgerichtet ist, für eine Volkswirtschaft, in der rund $10 \mathrm{v} . \mathrm{H}$. des Sozialprodukts im Außenhandel realisiert werden, mußte sich zur Erhöhung dieses Anteils viel bedeutenderer und weitreichender Maßnahmen als der Ausfuhrförderung und Einfuhrsubstitution durch inländische Erzeugnisse bedienen ${ }^{4}$.

1 In der jugoslawischen Handelsbilanz mit der UdSSR ergab sich 1967 bei einer Ausfuhr von 2 734, - Mill. neue Dinar und einer Einfuhr von 2 048, - ein Utberschuß von 686, - Mill. neuen Dinar.

Vgl. I. Zuvela, a.a.O., S. 517.

2 Als Beispiel für eine weitere Konkretisierung seien hier die komperativen Vorteile, die für die SR Kroatien festgestellt wurden, genannt: Seeschiffahrt, Schiffbau, Produktion von Grundnahrungsmitteln, Fremdenverkehr, petrochemische Industrie, Aluminiumindustrie, Produktion von Anlagen und Ausrüstungen für die Verfahrens- und Elektroindustrie, Holzveredelungsindustrie usw.

Vgl. Gesellschaftsplan der SR Kroatien für die Zeit 1966-70, Zagreb 1966, S. 42 .

${ }^{3} \mathrm{Vgl}$. Društveni plan razvoja Jugoslavije 1966-70 (Gesellschaftsplan der EntWicklung Jugoslawiens 1966-70), Beograd 1967, S. $83 \mathrm{f}$.

${ }^{4}$ Der Fünfjahresplan 1966-70 hat für die Außenwirtschaft folgende Ziele gesetzt: stärkere Einschaltung in die internationale Arbeitsteilung; konkurrenzfăhiges Angebot auf den Weltmärkten; langfristige Unternehmenspolitik in Produktion und Ausfuhr aufgrund der allgemein gültigen Kriterien Rentabilität und Wirtschaftlichkeit (also ohne Subventionen); Intensivierung der Wirt- 
1. Da der Außenhandel für die wirtschaftliche Entwicklung des Landes mehr an Bedeutung gewinnen sollte, mußten umfangreiche Maßnahmen ergriffen werden, die die politischen und administrativen Hindernisse auf dem Wege $z u$ einem intensiveren Austausch von Gütern und Dienstleistungen mit dem Ausland beseitigen sowie die Konkurrenzfähigkeit der jugoslawischen Wirtschaft auf den inländischen wie ausländischen Märkten heben sollen.

2. Zu dieser neuen Aufgabe kam noch eine weitere hinzu, nämlich die Rolle, die dem Außenhandel heute in der jugoslawischen Wirtschaftspolitik zukommt. Er übernahm die Korrektur der inländischen Preise, welche unter den Bedingungen spezifischer Marktformen in vielen Zweigen und Unternehmungen, insbesondere in der Industrie, gebildet wurden. Durch die Reform wurden zunächst die größten Differenzen beseitigt sowie das Preisniveau einiger Agrarerzeugnisse und industrieller Rohstoffe gehoben. Man mußte aber auch die monopolistischen Preise der inländischen Produzenten durch die ausländische Konkurrenz paralysieren. Deshalb hat man im Prinzip den Weltmarktpreis als Maßstab für die inländische Preisbildung verwendet, und zwar als konkreten, effektiven Preis für jugoslawische Erzeugnisse, die auf Weltmärkten gehandelt werden, also nicht als Durchschnittspreis.

Die erforderliche Erhöhung der Einfuhren, insbesondere für die Industrie, verlangte nach einer immer größeren Einschaltung in die internationale Arbeitsteilung auch auf der Seite der Ausfuhr. Da die Exporte hinter den Einfuhren zurückblieben, mußte man sie konkurrenzfähig machen, nicht nur bezüglich der Preishöhe, sondern auch in ihrer Preisstruktur sowie der Qualität. Für diesen Zweck war jedoch eine Liberalisierung auf den inländischen Märkten unerläßlich.

3. Die Produktionskapazităt der Industrieunternehmungen liegt häufig unter dem technologischen Rentabilitätsminimum und viele Kapazitäten werden ungenügend ausgenutzt. Es mußten größere Märkte für einige ausgewählte Industriezweige bzw. Unternehmungen geschaffen werden, um die bestehenden Kapazitäten voll zu nutzen.

Fortsetzung der Fußnote 4 von Vorseite:

schaftsbeziehungen zum Ausland durch eine Ausweitung des Güter - und Leistungsaustausches sowie einen Ausbau verschiedener Kooperationsformen; rational ausgeglichene regionale Ein- und Ausfuhrbilanzen; Herstellung des Gleichgewichts der laufenden Zahlungsbilanz; stärkerer Anstieg der Dienstleistungsexporte; Erweiterung und Diversifizierung der geschaftlichen Beziehungen jugoslawischer Unternehmungen mit ausländischen Partnern aufgrund von Kredit- und Kooperationsvereinbarungen; Herstellung der Konvertibilität des Dinars.

Vgl. Drustveni plan razvoja Jugoslavije 1966-70 (Gesellschaftsplan der Entwicklung Jugoslawiens 1966-70), Beograd 1967, S. 879 ff. 
4. Einer Erhöhung des Absatzes auf ausländischen Märkten standen nicht nur die Schwierigkeiten in der Produktion sowie der Erschließung ausländischer Märkte entgegen, sondern auch das bei den einheimischen Unternehmungen nicht in ausreichendem Maße vorhandene know how. $\mathrm{Zu}$ den exportierten Kapitalgütern müßte man nicht nur die notwendige technische Dokumentation sowie den Service, sondern auch die Methoden der Marktforschung mitliefern.

5. Die Außenhandelspolitik ist noch immer weit entfernt von den konkreten Möglichkeiten. Die Nationalbank als Hauptträger der Devisenpolitik befaßt sich nach wie vor hauptsächlich nur mit der verwaltungsmäßigen Erfassung der Zahlungsmittel sowie ihrer Redistribution zur Begleichung der Verbindlichkeiten aus dem zentralen Devisenfonds; - Mittel also, welche von jenen eingenommen werden, die diese Devisen verdienten und an jene verteilt werden, welche nicht in der Lage waren, sie zu e rwerben.

6. Schließlich erfordern die Integrationsbestrebungen in Europa eine Anpassung der jugoslawischen Wirtschaft an die neue Situation. Jugoslawien hat notwendigerweise ein großes Interesse, sich an dieses Europa zu binden. Es steht vor dem Problem, wie es sich den großen regionalen Integrationsräumen in Europa anschließen kann.

Jugoslawien hat auch die Frage zu lösen, in welcher Weise die Zusammenarbeit mit der dritten Welt, den Entwicklungsländern, die am jugoslawischen Außenhandel mit rund $12 \mathrm{v} . \mathrm{H}$. beteiligt sind, gestaltet werden soll.

Die Vereinigten Staaten als die größte Handelsmacht der Welt sind ebenfalls ein Faktor, der in der Wirtschaft Jugoslawiens seiner Bedeutung entsprechend berücksichtigt werden muß. Die USA stehen bei der jugoslawischen Einfuhr an erster Stelle (12 v. H. ) und bei der Ausfuhr an vierter Stelle (6, 1 v. H.). In genau entgegengesetzter Position befindet sich die Sowjetunion, welche bei der Ausfuhr mit 18,5 v.H. die erste Stelle und bei der Einfuhr mit 9, 3 v. H. die vierte Stelle einnimmt. (Vgl. Tabelle 6 s. Seite 17)

Die Wirtschaft Jugoslawiens ist gemäß ihrer Tradition, dem bisherigen Entwicklungstrend sowie dem gegenwärtigen Entwicklungsstand entsprechend, nach Europa ausgerichtet. Fast 80 v. H. ihres Außenhandels entfallen auf diesen Teil der Welt. 
Tab. 6: Der jugoslewische AuBenhandel mit den wichtigsten Handelspartnern 1961 und 1963-67 (Handelaumsatz in Mill. neue Dinar; 1 US $\$ 12,50$ neue Dinar)

\begin{tabular}{|c|c|c|c|c|c|c|c|c|c|c|c|c|}
\hline Position & 1961 & & 1963 & & 1964 & & 1965 & & 1966 & & 1967 & \\
\hline \multicolumn{13}{|c|}{ A us fuhr } \\
\hline 1 & Italien & 877 & Italien & 1971 & Italien & 1649 & UdSSR & 2344 & UdSSR & 2422 & Itallen & 2817 \\
\hline 2 & BR Deutachland & 722 & UdSSR & 1064 & UdSSR & 1456 & Itallen & 1824 & Italien & 2163 & UdSSR & 2734 \\
\hline 3 & UdSSR & 635 & BR Deutschland & 1019 & BR Deutachland & 963 & BR Deutechlend & 1158 & BR Deutachland & 1423 & BR Deutschland & 1208 \\
\hline 4 & Grosbritennien & 595 & USA & $\$ 76$ & DDR & 791 & DDR & 950 & USA & 936 & USA & 997 \\
\hline 5 & Polen & 504 & Grobbritannien & 543 & Grosbritannien & 745 & Thechechoslow akel & 888 & Tschechoslowakel & 845 & DDR & 787 \\
\hline 6 & USA & 457 & Polen & 476 & USA & $68 \mathrm{R}$ & USA & 836 & DDR & 805 & Tochechoslowakei & 627 \\
\hline 7 & DDR & 377 & DDR & 470 & Tachechoslow akel & 560 & Polen & 803 & Polen & 704 & Osterreich & 603 \\
\hline 8 & Osterreich & 319 & Oaterreich & 354 & Polen & 495 & Grobbritannien & 557 & Osterreich & 559 & Grobbritannien & 575 \\
\hline 9 & Griecheniand & 245 & Liberia & 323 & Osterreich & 364 & Indien & 371 & Grobbritannien & 549 & Polen & 573 \\
\hline 10 & Ungarn & 225 & Tschechoslowakei & i 290 & Indien & 352 & Oaterrelch & 326 & Indien & 535 & Ungern & 376 \\
\hline \multicolumn{13}{|c|}{ Elntuhr } \\
\hline 1 & USA & 2254 & USA & 2322 & Itallen & 2178 & USA & 2447 & USA & 2498 & BR Deutechland & 3575 \\
\hline 2 & BR Deutachland & 1781 & Italien & 1408 & USA & 2180 & Itallen & 1727 & Italien & 2106 & Itallen & 2849 \\
\hline 3 & Italien & 1560 & BR Deutechland & 1221 & BR Deutechland & 1393 & BR Deutschlend & 1432 & BR Deutschland & 1933 & UdSSR & 2048 \\
\hline 4 & Großbritannien & 575 & UdSSR & 907 & UdSSR & 1250 & UdSSR & 1343 & UdSSR & 1822 & USA & 1550 \\
\hline 3 & DDR & 470 & Grobbritannien & 627 & Großbritennien & 1041 & Großbritannien & 1106 & Trechechoslowakel & 1165 & Techechoslowakel & 1177 \\
\hline 6 & Ungarn & 410 & DDR & 612 & Tschechos low akel & $10: 7$ & Tschechoslowakei & 890 & DDR & 1099 & Grosbritannien & 1140 \\
\hline 7 & Osterrelch & 405 & Frankreich & 595 & DDR & $90 s$ & DDR & 794 & GroBbritannien & 1098 & Frankreich & 1009 \\
\hline 8 & UdSSR & 400 & Tachechoolowakel & 537 & Polen & 744 & Polen & 714 & Polen & 901 & DDR & 860 \\
\hline 9 & Polen & 357 & Polen & 412 & Frankreich & 644 & Frankrelch & 612 & Frankreich & 582 & Osterreich & 731 \\
\hline 10 & Frankreich & 294 & Onterreich & 396 & Ungarn & 446 & Osterreich & 434 & Japan & 495 & Polen & 519 \\
\hline
\end{tabular}

Quelle: Statiatike apol jne Irgovine Jugoslavije (Statistik des jugoslawischen AuBenhandels), Beograd, versch. Jahrgange. 
Es bestehen permanente Faktoren, die auf eine solche Orientierung Einfluß ausüben. Dies sind vor allem: die geringe räumliche Entfernung und die günstigen Handelswege; die technische Ausrüstung sowie Normung der jugoslawischen Industrie entsprechen vorwiegend der europäischen; in Europa befinden sich die Handelspartner Jugoslawiens, mit denen es seine traditionellen Güter tauscht; es werden dort Bedürfnisse für neue Produkte geweckt und neue Produkte kommen aus diesen Ländern; Rechtsnormen und Handelsbräuche entwickelten sich auf einer gemeinsamen europäischen Basis.

Es handelt sich demnach nicht nur um den reinen Handelsverkehr, der einen zufäligen, von der Konjunktur bedingten Charakter trägt, sondern um die internationale Arbeitsteilung, in die sich die jugoslawische Wirtschaft strukturell einfügt. Auch die im Zeichen der Reform des Außenhandels stehende Wirtschaftspolitik erkannte einen Vorteil in der Tatsache, daß auf westlichen Märkten eine kontinuierliche Versorgung mit Rohstoffen, Halberzeugnissen und Kapitalgütern in einem praktisch unbegrenzten Ausmaß möglich ist. Man weiß besonders zu schätzen, daß von dem vergleichsweise höheren Stand der technologischen Entwicklung ein enormer Ansporn für die eigene technologische Entwicklung und die Vermittlung von know how ausgeht. 1

Diese Tatsachen sind von grundlegender Bedeutung für die Durchführung der Reform in Richtung einer Modernisierung der Wirtschaft und Umwandlung ihrer Struktur.

Nicht weniger bemerkenswert ist die Tatsache, daß bei ungehinderter A rbeitskräftemobilităt ungefähr 300.000 jugoslawische Arbeiter - davon sind 60.000 Fachleute - Beschäftigung in den westeuropäischen Ländern gefunden haben, die mit ihren Utberweisungen zum Ausgleich der Zahlungsbilanz einen ansehnlichen Beitrag leisten.

In diesem Zusammenhang muß man auch den Fremdenverkehr, einen sehr dynamisch wachsenden Zweig der jugoslawischen Volkswirtschaft, erwähnen. Die bei weitem größte Zahl an Touristen sowie an Einnahmen aus dem Tourismus kommen aus Westeuropa.

Auch die Einnahmen aus dem internationalen Verkehr stammen größtenteils aus Westeuropa und dem offenen Weltmarkt; daran sind vor allem die Handelsmarine und der Transitverkehr beteiligt.

1 Den bescheidenen Entwicklungsstand der jugoslawischen Technik kann man daran erkennen, daß bis 1964: 341 Lizenzen von anderen Ländern erworben wurden und an das Ausland nur 39 Lizenzen, deren Gesamtwert jedoch bei 6 Mrd. Dinar lag, veräußert werden konnten.

Vgl. D. V. Cerović, Ugovori o licencame (Lizenzverträge), Beograd 1965, S. $39 \mathrm{f}$. 
Auffassungen, die sich gegen eine dauerhafte wirtschaftliche Zusammenarbeit wenden, entspringen ideologischen Motiven: So wird die Meinung vertreten, daß die westeuropäische Gemeinschaft eine Organisation sei, welche eine Ausbeutung mit Hilfe internationaler Kartelle betreibt. Dem steht jedoch die Tatsache entgegen, daß die Mehrheit jugoslawischer Gastarbeiter selbst, aus freiem Willen heraus, in diesem und einem solchen Europa arbeiten wollen.

Das zweite Gegenargument beruht auf der Eigenart der politischen Verhältnisse, welche für das sozialistische Jugoslawien z. T. unannehmbar sind. Die Furcht vor einer imperialistischen Politik bzw. der Einwirkung auf ein sozialistisches Land wie Jugoslawien, ist offensichtlich vorhanden, besonders in letzter Zeit und in einigen Kreisen. Unter den heutzutage herrschenden Kräfteverhältnissen in der Welt gibt es jedoch verschiedene Möglichkeiten zur Herstellung eines Gleich gewichts sowie zur Anbahnung einer internationalen Zusammenarbeit, und diese müssen nicht unbedingt ungünstig für Jugoslawien sein. Es besteht auch die Furcht, daß durch den Handel mit kapitalistischen Ländern kapitalistische Produktionsverhältnisse in Jugoslawien importiert werden.

Ein Gegenargument, das ich zu diesen Behauptungen erfuhr, läuft darauf hinaus, daß in einer solchen politischen Kombination mehrere zehn Millionen von außerordentlich bewußten und organisierten und dem Sozialismus zugeneigten Arbeitern leben, mit denen man sich einen gemeinsamen Aufbau der sozialistischen Zukunfï vorsteilen und ihrem Leiversstaridard beipflichten könnte.

Der dritte Einwand ist ein ökonomischer. Für Jugoslawien sei es schwer, eine den europäischen Verhältnissen vergleichbare und konkurrenzfähige Produktivität, Intensität und Rentabilität der Produktion zu erreichen; demzufolge sei es vorteilhafter, sich auf die Dauer den industriell weniger entwickelten Ländern zuzuwenden, denen gegenüber man größere relative Vorteile besitzt.

Die Antwort auf diese Argumentation ist, daß man von einer dauerhaften Verbindung nur dann sprechen kann, wenn gleichzeitig eine langfristige Anpassungszeit von zwanzig und mehr Jahren gegeben ist, wie dies das Beispiel Griechenlands und der Türkei zeigt. Ferner würde eine Bindung an ökonomisch und technisch schwächer entwickelte Länder wie eine Bremse auf die technologische Entwicklung Jugoslawiens wirken und seine Stellung in der Weltwirtschaft schwächen ${ }^{1}$.

1 Charakteristisch für die Beurteilung des technischen Fortschritts ist, daß bis 1964 vom Westen 312, vom Osten jedoch nur 29 Lizenzen erworben wurden.

Vgl. D. V. Cerović, a.a.O., S. 48. 
Jugoslawien hat sehr gute, aber nicht formalisierte Beziehungen zur EFTA.

Bei der Europäischen Wirtschaftsgemeinschaft (EWG) hat Jugoslawien bereits Anfang 1962 um Verhandlungen nachgesucht und diesen Antrag einige Male wiederholt. Es scheint jedoch, als ob es erst in diesem Jahr (1968) zu Verhandlungen zwischen der EWG und Jugoslawien kommen wird. Wenn auch diese Gespräche ihrem Charakter nach Verhandlungen über aktuelle Handelsprobleme sind, so könnten sie dennoch einen Weg für langfristige Lösungen aufzeigen. Probleme, die es zu lösen gilt, sind politischer wie wirtschaftlicher Natur.

Meiner Meinung nach würden - wenn man davon ausgeht, daß die EWG vornehmlich eine politische Organisation ist - bindende Vereinbarungen vor allem von politischen Faktoren abhängen, u.a. auch davon, in welchem Ausmaß die EWG ihre Türen für andere Länder öffnet. Daher sollte man die Entwicklung genau verfolgen, wie die politische Problematik bei der Aufnahme neutraler Länder (wie Schweden, die Schweiz oder Österreich) gelöst wird, um dann die Position Jugoslawiens bestimmen zu können.

Vom ökonomischen Standpunkt aus gesehen ist es klar, daß der Prozeß einer Anpassung Jugoslawiens an die EWG ein langfristiger Prozeß sein muß, vielleicht etwas kürzer als der bei einigen wirtschaftlich weniger entwickelten europäischen Ländern, die mit der EWG bereits vertragliche Beziehungen unterhalten.

Unserer Meinung nach sollte Jugoslawien keine Zeit verlieren, sondern sich schon vor Abschluß eines formellen Vertrages a uf die strukturellen Umstellungen vorbereiten. Diese Umstellung ist bei der Aufnahme einer institutionalisierten langfristigen Zusammenarbeit unerläßlich. Sie würde außerdem für unser Land eine günstige Ausgangsposition schaffen.

Diesem Zweck dienen u.a. (1) autonome, allein die Binnenwirtschaft betreffende Maßnahmen zur Erhöhung der Konkurrenzfähigkeit der jugoslawischen Wirtschaft auf dem Weltmarkt und (2) Bemühungen um den Abschluß langfristiger Kooperations- und Koproduktionsverträge mit Unternehmungen in den Ländern des Gemeinsamen Marktes, die zweifelsohne einen Anschluß Jugoslawiens an diesen Markt erleichtern würden.

Jugoslawien und das COMECON

Das Verhältnis zum COMECON ist so geartet, daß Jugoslawien (Beobachter seit 1956) seit 1965 ein besonderes, für das Land günstiges Arrargement besitzt. Es kann sich an bestimmten Vorhaben dieser Organisation 
beteiligen, falls man sich aufgrund autonomer Entscheidung davon Erfolge verspricht.

Über den weiteren Ausbau einer Zusammenarbeit mit dem COMECON bestehen unterschiedliche Auffassungen ideologischer, technologischer und wirtschaftlicher Motivierung. Die vornehmlich dogmatisch orientierten Kreise stellen ideologische Motive in den Vordergrund. Sie sehen im COMECON eine Organisation, die geeignet ist, die internationale sozialistische Arbeitsteilung auszubauen sowie eine kommunistische Zukunft zu verwirklichen. Die Ziele müßten - so wird argumentiert - unter ideologisch-politischen und supranationalen Gesichtspunkten vom COMECON festgelegt werden, wobei Jugoslawien seine ökonomischen Interessen diesen Zielsetzungen unterzuordnen habe.

Eine zweite Gruppe, die technologische Motive in die Diskussion wirft, hebt folgende Vorteile einer Zusammenarbeit im COMECON hervor:

- Jugoslawien könnte im Wege einer Ausweitung seiner Handelsbeziehungen mit den zentralgeleiteten Planwirtschaften des Ostens seine brachliegenden Kapazitäten ausnutzen sowie die bestehenden strukturellen Schwierigkeiten überwinden;

- Die künftigen wirtschaftlichen Beziehungen innerhalb eines sozialistischen Weltmarktes müßten durch vertragliche Abmachungen mit den Planwirtschaften Osteuropas vorbereitet werden. Zwar seien diese Länder im Ausbau solcher Beziehungen außerordentlich schwerfällig, könnten jedoch diesen Nachteil durch die einem langfristig geplanten Handel innewohnende Sicherheit ausgleichen.

Schließlich wäre noch eine dritte Gruppe zu nennen, die in einer Zusammenarbeit mit dem COMECON kommerzielle Interessenvorteile erblickt. Bekanntlich bestehen auf den osteuropäischen Märkten hohe Preise für Konsumgüter; Jugoslawien könnte für seine Erzeugnisse der Konsumgüterindustrie dort günstige Preise erzielen und einer Umstrukturierung seiner Wirtschaft aus dem Wege gehen.

Die Zurückhaltung, die bei der Beurteilung eines weiteren Ausbaus der Beziehungen zum COMECON und seinen Mitgliedsstaaten erkennbar ist, beruht auf politischen und ökonomischen Argumenten:

1. Man befürchtet eine rezidive Politik des "großen Bruders".

2. Die bisherigen Erfahrungen einiger Vollmitglieder des COMECON veranlaßten diese, ihre Beziehungen zu der Organisation nur bis zu einer bestimmten Grenze auszuweiten.

3. Ferner ist man der Meinung, daß eine vollständige oder überwiegende Bindung an das COMECON zur Absonderung vom Weltmarkt führen und 
die Handelsbeziehungen unter den Bedingungen der internationalen Arbeitsteilung einschränken könnte. Die in einem Sektor günstigen Preise werden in Wirklichkeit durch ungünstige Preise in einem anderen kompensiert. Zudem werden in absehbarer Zeit die noch vorhandenen Preisvorteile durch die internationale Konkurrenz und den steigenden Output in diesen Ländern selbst allmählich abgebaut.

4. Außerdem glaubt man, im Handel mit den zentralgeleiteten osteuropäischen Volkswirtschaften mit starrer Planwirtschaft Gefahren für die eigene Dezentralisierungspolitik und Demokratisierung zu erkennen (dies speziell wegen des zentralisierten An- und Verkaufs in den osteuropäischen Ländern mit staatlichem Außenhandelsmonopol auf Makround Mikroebene), d.h. es könnten staatssozialistische Verhältnisse importiert werden.

5. Vor allem eine Absonderung vom Weltmarkt wäre sehr gefährlich, da dies eine Anpassung der inländischen Preise an die Bedingungen der internationalen Arbeitsteilung verhindern und den technologischen Prozeß ungenügend stimulieren würde, was doch in der Regel beim Fehlen einer internationalen Konkurrenz der Fall ist. Viele zentral und autoritativ geplanten Volkswirtschaften sind außerordentlich langsam und einseitig in der Einführung des technischen Fortschritts.

6. Die Abwicklung des Zahlungsverkehrs auf bilateraler Clearingbasis stellt entweder von Haus aus ein Hindernis für die Entwicklung gegenseitiger Beziehungen dar, oder dieses Hindernis tritt auf, wenn antizipierte Nachfrage in Form nicht ausgeglichener Zahlungsbilanzüberschüsse entsteht, so daß zwangsweise zum Ausgleich der Bilanz Güter importiert werden müssen, auch dann, wenn dies den wirtschaftlichen Interessen Jugoslawiens widerspricht.

Kurzum überwiegt die Auffassung, daß die Reform, die eine Stärkung der Konkurrenzfăhigkeit Jugoslawiens auf dem Weltmarkt herbeiführen will, den jugoslawischen Außenhandel von den engen Grenzen der administrativen Autarkie loslösen und ihn auf weltweiter Basis konkurrenzfähig machen müßte. Den Handel mit den sozialistischen Ländern muß man als eine Art von Wirtschaftsbeziehungen ansehen, durch die man mit internationalen Maßstäben Erfolg und Nutzen gegenseitiger Beziehungen messen kann. Mit anderen Worten, man sollte mit den Ländern Osteuropas wie auch mit der übrigen Welt langfristige Abkommen schließen ${ }^{2}$.

1 In diesem Sinne kann man auch die wirtschaftspolitische Entschließung des $\mathrm{ZK}$ des BdKJ interpretieren, die keiner regionalen Gruppierung den Vorrang einräumt und wo es heißt: "Es ist gleichfalls notwendig, größere Anstrengungen zu unternehmen, um günstigere Bedingungen im Handel mit den regionalen Wirtschaftsgruppen sowie den Entwicklungsländern zu schaffen".

Vgl. "Vjesnik", Zagreb, v.26.11.1967. 
Wirtschaftliche Beziehungen zu den Entwicklungsländern

In den wirtschaftlichen Beziehungen $\mathrm{zu}$ den weniger entwickelten Ländern ist man in Jugoslawien nach einer langen Zeit, die vom politischen Optimismus erfüllt war und durch teure Erfahrungen bezahlt wurde, zu einem Stadium - so glauben wir - realistischer Beurteilung gelangt; einerseits in der Abschätzung des vorhandenen Potentials und des Entwicklungstempos in einigen Ländern, andererseits in der Beurteilung der Möglichkeiten Jugoslawiens, sich an diesem Prozeß zu beteiligen.

Neben den allgemein bekannten Bedingungen in den wirtschaftlichen Beziehungen $z$ den Entwicklungsländern (wie z. B. hohe fiskalische Belastungen, Schwierigkeiten beim Zahlungsbilanzausgleich usw.) besitzt Jugoslawien spezifische Vor- und Nachteile hinsichtlich einer Bewäligung dieser Probleme.

Vorteile sehen wir in folgenden Tatsachen:

1. Die von den Entwicklungsländern ausgehende Nachfrage kann zu einer Beschleunigung der jugoslawischen Industrialisierung beitragen, wobei sich die Effekte des Akzelerationsprinzips nachhaltig auswirken können ${ }^{1}$. Hinzukommt, daß sich der Bedarf in den Entwicklungsländern nicht so sehr vom jugoslawischen unterscheidet. Jedoch tritt Jugoslawjen als hochentwickeltes Land diesen Ländern gegenüber, was zur Folge hat, daß es entsprechend der Ausfuhrstruktur seine traditionellen Exportzweige aufgeben könnte.

2. In der Aufgeschlossenheit für die Bedürfnisse der Entwicklungsländer, entsprechend der jugoslawischen Politik der Nichtverpflichtung (was man vor allem $z$. B. bei der Formulierung der Politik der UNCTAD I und II bemerken kann). Diesem günstigen politischen Klima entspricht nicht immer eine adäquate Handelsorganisation oder entsprechende Anstrengungen in der Produktion. Andererseits hatte das günstige politische Klima bei den Entwicklungsländern (insbesondere in den ersten Jahren ihrer Selbständigkeit) unbegründete Erwartungen hervorgerufen, was unökonomische Momente in die Handelsbeziehungen und die Erfüllung der übernommenen Verpflichtungen hineintrug.

3. Der dritte Vorteil liegt in der günstigen Lage Jugoslawiens als Küstenland mit einer eigenen Handelsmarine.

Diesen vorteilhaften Tatsachen muß man die nachteiligen Momente gegenüberstellen:

1 Wir beziehen uns vor allem auf die erfolgreiche Tätigkeit jugoslawischer Unternehmungen bei Bau von Häfen und Kraftwerken, bei geologischen Forschungsarbeiten usw. 
1. In Jugoslawien fehlt es oft an ausreichenden Kenntnissen über die betreffenden Absatzmärkte sowie an einer effektiven Absatzorganisation, so besonders im Handel mit den überseeischen Entwicklungsländern.

2. Die Empfindlichkeit dieser Märkte sowie die auf innen ausgetragene heftige internationale Konkurrenz wurden oft unterschätzt.

3. Die Kreditkapazität Jugoslawiens ist unzureichend, insbesondere zum Export von Maschinen, Ausrüstungen und dauerhaften Konsumgütern. Bisher hat Jugoslawien an Ausfuhrkrediten einen Betrag von über 800 Mill. Dollar aufgewendet, was für das Land eine beträchtliche Belastung darstellt. Das macht deutlich, daß die potentiellen Absatzchancen in den Entwicklungsländern für Jugoslawien größer sind, als seine gegenwärtigen Möglichkeiten. Diesen entgangenen Gewinn muß man empfindlich spüren.

Aufgrund unserer Erfahrungen mit den Wirtschaftsbeziehungen zu weniger entwickelten Ländern sind wir zu dem Ergebnis gekommen, daß man

1. außerökonomische Motivierungen absondern und nach strengen ökonomischen Grundsätzen vorgehen sollte, d.h. jene Beziehungen entwickeln, welche zur Erhöhung des jugoslawischen Volkseinkommens beitragen, wobei das natürlich nicht bei jeder einzelnen Transaktion der Fall sein muß;

2. bestrebt sein sollte, diese Beziehungen stabiler zu gestalten, d.h. ihnen einen langfristigen Charakter $z$ verleihen;

3. Umfang, Intensität und Methoden der gegenseitigen wirtschaftlichen Beziehungen je nach dem erreichten Entwicklungsstand des betreffenden Landes differenzieren soll te.

A usblick

Wollen wir in kurzen Zügen theoretisch und logisch die Problematik der wirtschaftlichen Beziehungen Jugoslawiens zu Ost und West, zu weniger entwickelten und hochentwickelten Volkswirtschaften klar herausstellen, so kann das Dilemma vom Standpunkt der langfristigen wirtschaftlichen Entwicklung wie folgt formuliert werden:

Mit den hochentwickelten Ländern tauscht Jugoslawien seine traditionellen Ausfuhrguiter, wie Agrarerzeugnisse und Rohstoffe, gegen den technischen Fortschritt auf hohem Niveau. In die weniger entwickelten Länder exportiert Jugoslawien seine neuen Industrieerzeugnisse und konserviert dadurch den gegenwärtigen Industrialisierungsproze $\beta$ in seiner Struktur. 
Das Problem, dessen Lösung in der Zukunft liegt, ist, in welchem Maß es Jugoslawien gelingen wird, sich mit seinen Industrieerzeugnissen und den neuen Produkten auf den Märkten der Industrienationen durchzusetzen, um auf diese Weise den technischen Fortschritt für sich zu sichern, bzw. inwieweit der technische Fortschritt in den weniger entwickelten Ländern eine Umwandlung der jugoslawischen Wirtschaftsstruktur fördern kann, was allerdings gleichbedeutend mit einem Zurückbleiben hinter dem weltwirtschaftlichen Fortschritt wäre. Einstweilen sind die jugoslawischen Exporte in die westlichen und östlichen Länder dem Umfang nach fast gleich groß, doch liegen die Importe aus den westlichen Ländern bedeutend höher.

Diese Problematik hat ihre politische wie ökonomische Seite.

Politisch gesehen hängt alles davon ab, inwieweit der Welthandel ein offener Prozeß bleibt und die regionalen Gruppen gegenüber Jugoslawien offen sein werden.

Die ökonomische Seite der Problematik sehen wir darin, inwieweit der Konkurrenzkampf zu monopolistischen Verhaltensweisen oder zur Anwendung planwirtschaftlicher Mechanismen in der Wirtschaftspolitik führen wird; andererseits aber auch darin, in welchem Maße die Starrheit der Planung durch die Einführung marktwirtschaftlicher Mechanismen aufgelockert und der Markt von administrativen Schranken befreit wird.

Es hat sich bisher erwiesen, daß in den hochentwickelten Ländern elastische Marktkräfte existieren, die, was die gegenseitige wirtschaftliche Zusammenarbeit anbelangt, viel effizienter waren, als die starren planwirtschaftlichen Systeme, die vorwiegend auf national-autarkiepolitischen Konzeptionen beruhen.

Wir sind der Ansicht, daß die technische Revolution des 20. Jahrhunderts eine monopolistisch strukturierte Marktwirtschaft zwangsläufig zur Anwendung der Planung drängt. Gleichzeitig aber zwingt diese technische Revolution die zentrale und autoritative Planung eines Landes zur Nutzbarmachung marktwirtschaftlicher Mechanismen. Dieser konvergierende Prozeß zeigt sich gewissermaßen in Jugoslawien.

Aber ist denn dieses Dilemma so bedeutend und dominierend, daß man es heute zum entscheidenden Faktor der jugoslawischen Politik erhebt? In der Realität gibt es noch eine Reihe anderer Faktoren, welche die Beziehungen Jugoslawiens zu wirtschaftlich stärker oder schwächer entwickelten Ländern bestimmen.

So kann man zum Beispiel als Folge der stetig zunehmenden Integrationsbewegungen in der Welt, besonders aber in Europa, in naher Zukunft die Zeit voraussehen, in der zwei große regionale Gruppen, die im Osten und Westen, beginnen werden, gemeinsame Verhandlungen zu führen und langfristig ihre ökonomischen Beziehungen zu regeln. Insofern könnten die von 
Jugoslawien gemachten Erfahrungen eine bedeutende Rolle spielen, und zwar nicht nur auf diplomatisch-politischem Gebiet. Wir denken hierbei auch an die Gewinnung praktischer Erfahrungen in der unmittelbaren $\mathrm{Zu}$ sammenarbeit mit Partnern unterschiedlicher Wirtschaftssysteme ${ }^{1}$.

In dieser Beziehung sind die neuesten wirtschaftspolitischen Maßnahmen Jugoslawiens zur Beteiligung ausländischen Kapitals an unserer Wirtschaft von besonderem Interesse.

Die Beteiligung ausländischen Kapitals

Bereits früher strömte ausländisches Kapital nach Jugoslawien in einem etwas größeren Umfang als in andere sozialistische Länder. Zum größten Teil waren es staatliche Kredite bzw. Anleihen anderer Länder sowie internationaler Organisationen ${ }^{2}$. Außerdem wurden seitens ausländischer Unternehmungen bzw. Banken zweckgebundene Kredite für die Anschaffung von industriellen Ausrüstungen, Lizenzen sowie für Kooperationsgeschäfte gewährt. Im Falle von Anleihen mußten Zinsen bezahlt werden, bei Lizenzen die vereinbarte Gebühr und bei der Kooperation waren es Erzeugnisse, mit denen man die Leistungen des Partners entschädigte.

Ein solcher Kapitalzufluß hatte jedoch nicht den gewünschten Nutzeffekt. Dieses Kapital kam ins Land aufgrund von Staatsverträgen oder unter staatlicher Absicherung, wobei Garantien für die Bonität des Schuldners sowie den Gewinntransfer vorhanden waren. Die einheimische Vertragspartei (Kapitalimporteur) war nicht ge zwungen, sich um eine optimale Mittelverwendung zu kümmern, auch nicht um die Beschaffung von ausländischen Zahlungsmitteln durch die Ausfuhr, denn dies war Sache des jugoslawischen Staates bzw. der Nationalbank, welche zu diesem Zweck die Distribution aller Devisen zentral vornahm.

Dem ausländischen Kapitalgeber war es somit ziemlich gleichgültig, wer Empfänger der Mittel war, wie dieser sie investierte und wie er sie nutzte, denn er hatte seinerseits eine staatliche Bürgschaft.

1 Wir sind überzeugt, daß man z. B. bei den Vereinbarungen über die Erstellung von Automobilfabriken in einem großen sozialistischen Land die Erfahrungen hätte verwerten können, welche sein Vertragspartner in der jugoslawischen Praxis bereits gemacht hat.

${ }^{2}$ Vgl. A. ¿ i ¿in-Sain, U. Dujకin, Kompleksno ukljuとivanje Jugoslavije u medjunarodnu podjelu rada - novi pristup (Die komplexe Eingliederung Jugoslawiens in die internationale Arbeitsteilung - neue Behandlung), Ekonomske studije, H. 4, Hrsg. vom Ekonomski Institut SRH, Zagreb 1967, S. 13 ff. Diese interessante und nützliche Studie ist für mehrere Stellen in dieser Arbeit herangezogen worden. 
Infolgedessen zeigte sich folgende Wirkung:

1. Es wurden nicht jene Kapitalgüter importiert, die man unter optimalen Gesichtspunkten hätte investieren können, sondern solche, für die man einen garantierten Kredit erhalten konnte. Solche Kredite wurden größtenteils für große Geschäftsabschlüsse, so z. B. für die Anschaffung kompletter neuer Produktionsstätten gewährt, während für die Modernisierung der vorhandenen Anlagen und die Beschaffung einzelner Maschinen keine Mittel vorhanden waren.

2. Der Kapitalzufluß war mehr von momentanen politischen Situationen im In- und Ausland abhängig, als an einer ökonomischen Wirtschaftsweise orientiert.

3. Dieses System der Kapitalbeschaffung stand im großen und ganzen im Widerspruch zur Wirtschaftspolitik des Landes, die auf eine Dezentralisierung und die Modernisierung der bestehenden Industrieunternehmungen ausgerichtet war.

4. Diese Verfahrensweise war sehr kostspielig, denn man differenzierte nicht genügend $z w i s c h e n$ den einzelnen Transaktionen. Der Kapitalzustrom hing im wesentlichen von der Entscheidung der Zentrale ab. Diese wiederum war bedingt durch die allgemeine Stellung des Staates als Ganzes in der Weltwirtschaft und die ganze Kette des Zustroms ausländischer Mittel war abhängig vom schwächsten Glied in dieser Kette (d. hi. vom größten Risiko) und Jugoslawien hatte daher auch die schlechtesten Konditionen.

Für den Übergang zur Möglichkeit einer unmittelbaren Beteiligung ausländischen Kapitals gibt es tiefere Gründe. Jugoslawien erreichte in seiner Entwicklung eine Stufe, die durch den Übergang von einer unterentwickelten zu einer entwickelten Wirtschaft gekennzeichnet ist. Es trat eine Differenzierung der Unternehmungen, Regionen und Strukturen ein.

Die Vorteile einer vertragsmäßigen direkten Investition ausländischen Kapitals in den jugoslawischen Unternehmungen sind folgende:

1. Bei einer direkten Beteiligung wird auch der ausländische Investitionspartner unmittelbar an der Rentabilität der Investition interessiert sein, zumal Aussichten auf einen gemeinsamen Gewinn sowie die Minderung des gemeinschaftlichen Risikos bestehen.

2. Der ausländische Investitionspartner wird eine fortschrittlichere Technologie, das know how sowie die Ausrüstung und das Marketing einbringen.

3. Der ausländische Partner wird auch einen Teil der Verantwortung für den Absatz der Erzeugnisse auf ausländischen Mărkten übernehmen, mit dem Ziel, die Deviseneinnahmen zu erhöhen und damit den Transfer 
seines Gewinnanteiles zu ermöglichen; auf diesem Wege kann auch die jugoslawische Gesamtausfuhr erhöht werden.

4. Er wird ferner von sich aus eine Rationalisierung in der Unternehmung, in welcher er seine Mittel investiert hat, vorantreiben (Leitung und Organisation der Geschäftstätigkeit).

5. Dadurch werden verschiedene Engpässe bei den bestehenden Unternehmungen beseitigt und die Modernisierung der jugoslawischen Industrie vorangetrieben.

Oben hatten wir bereits festgestellt, welche Wirtschaftszweige im Fünjahresplan genannt wurden, die komperative Vorteile gegenüber anderen Zweigen aufweisen. Grundsätzlich und vom Standpunkt eines Nationalökonomen gesehen, kann man die Bereiche, die sich für eine ausländische Beteiligung eignen, wie folgt bestimmen:

1. Die Produktion von Erzeugnissen, die bereits auf ausländischen und inländischen Märkten nachgefragt werden, deren Nachfrage aber nicht befriedigt werden kann, weil das inländische Kapitalangebot dafür $z u$ knapp ist. Ein Beispiel bietet der Fremdenverkehr an der jugoslawischen Adria.

2. Zweige, bei denen ein potentielles Faktorangebot vorhanden ist, es aber an unmittelbaren Kapitalquellen fehlt, um dieses Angebot auszunutzen bzw. die vorhandenen natürlichen Produktionsfaktoren zu erschließen. Ein Beispiel dafür bietet die Erzeugung von Bauxit und Aluminium sowie die Förderung von Rohöl an der Küste. Hier müßten auch Zahl und Qualităt der Arbeitskräfte sowie die Ausbildung von Fachleuten erwähnt werden.

3. Bereiche, wo ein Produktions- und Absatzmarkt besteht, das Produkt aber in kleineren Serien hergestellt wird und nicht kontinuierlich genug, um den Markt zu erschließen und, was noch wichtiger ist, einen schon erschlossenen Markt zu sichern. Beispiel: Ausfuhr von Haushaltsgegenständen aus Holz.

4. Dort wo eine Produktion und Märkte im Inland zwar vorhanden sind, die Produktion aber zu veraltet oder zu teuer ist, für eine Kostenminderung jedoch die nötigen Investitionsmittel fehlen. Ein Beispiel dafür bietet die Metall- und Maschinenindustrie.

5. Mit Hilfe von ausländischen Investitionen kann man in der Produktion auch auf neue Erzeugnisse sowie neue Verfahren übergehen, für diese einen eigenen inländischen oder andere Märkte ins Leben rufen, bei deren Erschließung bzw. Ausbau sich Jugoslawien eine gewisse Vorzugsstellung sichern könnte. 
Einige Wirtschaftszweige sind durch Gesetz ausdrücklich von der Möglichkeit einer Beteiligung ausländischen Kapitals ausgeschlossen, und zwar Banken, Versicherungen, Binnenverkehr, Binnenhandel, gebietskörperschaftliche und gesellschaftliche Dienstleistungen usw.

Der Form nach ist die direkte Investition ausländischer Kapitalmittel nur durch einen Vertrag sui generis möglich. Es handelt sich also weder um aktienrechtliche Beziehungen, noch um sogenannte Partnerships oder um die Gründung gemischter Gesellschaften. Bisher gab es im Geschäftsverkehr mit ausländischen Firmen ausschließlich nur die Möglichkeit des Anund Verkaufs, der Erlangung industriellen Eigentums sowie der Firmenvertretung, alles Formen, bei denen jeder Vertragspartner sein eigenes Interesse und sein eigenes $R$ isiko zu vertreten hatte.

Nun handelt es sich um vertraglich fixierte gemeinsame Interessen sowie um eine gemeinsame Risikotragung ${ }^{1}$. Dies ist nur deshalb möglich, weil die Unternehmungen in Jugoslawien wirtschaftlich und rechtlich unabhängig sind, selbst Entscheidungskompetenzen haben, sich auf den Märkten nach den Marktpreisen orientieren, selbst Arbeiter einstellen und entlassen, selbst ihre Pläne erstellen und beschließen, Pläne, die nur einen indikativen Charakter besitzen und keine rechtsverbindlichen Normen hinsichtlich der laufenden Geschäftstätigkeit enthalten.

Die Verträge über eine Beteiligung müssen drei Grundbedingungen beachten: gesellschaftliches Eigentum an den Mitteln der Wirtschaftsorganisation, Arbeiterselbstverwaltung und Eintragungspflicht, wenn bestimmte formelle und materielle Voraussetzungen erfüllt sind. Die wichtigsten sind: (a) der Anteil des ausländischen Kapitals darf in der Regel 50 v. H. des Gesamtkapitals der Unternehmung nicht übersteigen (davon können Ausnahmen gemacht werden); (b) es müssen die Voraussetzungen erfüllt sein, unter denen eine solche Zusammenarbeit in den internationalen Beziehungen stattfinden; (c) der Gleichheitsgrundsatz in Bezug auf die einheimische Wirtschaft und die inländischen Unternehmungen darf nicht verletzt werden. Dies sind die Voraussetzungen für eine Eintragung in das Register. Die Praxis wird es zeigen, inwieweit sie erfüllt werden können.

Die Unternehmung wird von einem Arbeitsrat geleitet. Die Verfügung über die Mittel des ausländischen Kapitalgebers wird durch Vertrag geregelt, durch den auch ein Geschäftsführungsrat eingesetzt und seine Zuständigkeit bestimmt wird.

Der ausländische Kapitalgeber haftet für Verbindlichkeiten bis zur Höhe seines Kapitalanteils und ist von der Haftung für alle anderen Verpflichtungen der jugoslawischen Unternehmung ausgeschlossen.

$1 \mathrm{Vgl}$. A. Gold $\mathfrak{y}_{\mathrm{tajn}} \mathrm{n}$, Ugovor o ulaganju inozemnih firmi u domaća preduzeća (Vertrag über die Einlagen ausländischer Firmen in den einheimischen Unternehmungen), Beograd 1967, S. $65 \mathrm{ff}$. 
Die Garantie für seine Einlage übernimmt die Unternehmung entsprechend den geltenden privatrechtlichen Bestimmungen. Der Staat seinerseits hat ein Recht souveräne Entscheidungen zu treffen, ist aber gehalten, die Normen und Prinzipien des internationalen Rechts zu beachten.

Bisher kann nicht sehr viel über Vertragsabschlüsse nach diesem Gesetz gesagt werden. Es sieht einstweilen so aus, als ob Inländer wie Ausländer abwarten, welche Formen sich entwickeln und welche Erfahrungen aus den ersten Schritten gewonnen werden können. Dies ist in der heutigen Situation verständlich, doch sind hier die Aussichten noch offen ${ }^{1}$.

Ich bin der Ansicht, daß diese Neuheit im jugoslawischen Wirtschaftssystem von großer Bedeutung ist, nicht allein für Jugoslawien, sondern von weltweiter Bedeutung, denn es befinden sich in einem ähnlichen Stadium wirtschaftlicher Entwicklung auch andere sozialistische Länder, so daß dadurch weitere Möglichkeiten einer friedlichen Zusammenarbeit aufgezeigt werden, die zur weltweiten Einführung des technischen Fortschritts sowie zur Schaffung potentieller Partner im internationalen Güteraustausch führen können.

Bayeriache

Bubtshis'otriek

Munchen

1 Nach Ansicht jugoslawischer Juristen sind noch folgende Fragen offen geblieben, die einer eingehenden Präzisierung bedürfen: Gewinntransfer, Repatriierung der ausländischen Kapitalanteile, technisches Management, Rolle des Geschäftsführungsrates, Verhältnis zu den Organen der Arbeiterselbstverwaltung, Möglichkeit und Einfluß des ausländischen Partners bei der Entscheidungsbildung. Auswahl der fachlichen Leitungsgremien.

Vgl. Interview mit A. Gold $\mathrm{stajn}_{\mathrm{j}}$, "Nedeljne Informativne Novine", Beograd, vom 14.1.1968. 


\section{Veroffentlichungen der Südosteuropa-Gesellschaft}

Verlagsauslieferung: Dr. Dr. Rudolf Trofenik, Südostbuchhandel KG, München 13, Elisabethstraße 18

Reihe SUDOSTEUROPA - J A R R B CH

1. Band: SÜDOSTEUROPA ZWISCHEN OST UND WEST - München 1957. 224 S., Ganzleinen DM 15,80

2. Band: IDEOLOGISCHE, KULTURELLE UND WIRTSCHAFTLICHE WANDLUNGEN IN SÜDOSTEUROPA - München 1958. $199 \mathrm{~S} .$. Ganzleinen DM 15,80

3. Band: WIRTSCHAFTLICHE ENTWICKLUNG UND VOLKLICHE EIGEN STÄNDIGKEIT IN SULDOSTEUROPA - München 1959. $245 \mathrm{~S} .$, Ganzleinen DM 15,80

4. Band: DER GEGENW ÄRIGE STAND DER WIRTSCHAFTLICHEN UND KULTURELLEN BEZIEHUNGEN ZU SUDOSTEUROPA - MÜnchen 1960. 191 S., DM 15,80

5. Band: DIE DONAU IN IHRER GESCHICHTLICHEN, W IRTSCHAFTLICHEN UND KULTURELLEN BEDEUTUNG - München 1961 . 187 S., Ganzleinen DM 15,80

6. Band: DIE VOLKSKULTUR DER SUDOSTEUROPAISCHEN VÖLKER München 1962. 216 S., Ganzleinen DM 18,-

7. Band: DEUTSCH -SÜDOSTEUROPÄISCHE WIRTSCHAFTSPROBLEME München 1966. 138 S., Ganzleinen DM 32,-

Reihe SUDOSTEUROPA-SCHRIFTEN

1. Band: VÖLKER UND KULTUREN SÜDOSTEUROPAS - Kulturhistorische Beiträge - München 1959. 284 S., broschiert DM 22, -

2. Band: WIRTSCHAFT UND GESELLSCHAFT SÜDOSTEUROPAS - Ge denkschrift für Wilhelm Gülich - München 1961. $600 \mathrm{~S}$., Ganzleinen DM 48,-

3. Band: AUFSÄTZE UND A BHANDLUNGEN ZUR GESCHICHTE SÜDOST EUROPAS UND DER LEVANTE I von Franz Babinger - München 1962. 474 S., Abb., Ganzleinen DM 56,-

4. Band: WIRTSCHA FTSW ISSENSCHA FTLICHE SÜDOSTEUROPAFOR SCHUNG - Grundlagen und Erkenntnisse - Ausgewählt und redigiert von Theodor Zotschew - München 1963. 280 S., Ganzleinen DM 36. - 